\title{
Optimal Timing of Single-Stage Retrograde Endoscopic Common Bile Duct Stone Removal in Mild and Moderate Acute Cholangitis: A Prospective Trial
}

\section{Chih-Ming Liang}

Chang Gung Memorial Hospital Kaohsiung Branch

\section{Yi-Chun Chiu}

Chang Gung Memorial Hospital Kaohsiung Branch

\section{Lung-Sheng Lu}

Chang Gung Memorial Hospital Kaohsiung Branch

\section{Cheng-Kun Wu}

Chang Gung Memorial Hospital Kaohsiung Branch

\section{Fai-Meng Sou}

Chang Gung Memorial Hospital Kaohsiung Branch

Pao-Yuan Huang

Chang Gung Memorial Hospital Kaohsiung Branch

\section{Te-Ling Ma}

Chang Gung Memorial Hospital Kaohsiung Branch

\section{Chung-Huang Kuo}

Chang Gung Memorial Hospital Kaohsiung Branch

\section{Seng-Kee Chuah}

Chang Gung Memorial Hospital Kaohsiung Branch

Chung-Mou Kuo ( $\sim$ kuo51116@gmail.com )

Chang Gung Memorial Hospital Kaohsiung Branch

\section{Research article}

Keywords: cholangiopancreatography, endoscopic papillary balloon dilation, endoscopic retrograde endoscopic sphincterotomy, post-endoscopic retrograde cholangiopancreatography pancreatitis

Posted Date: June 22nd, 2020

DOI: https://doi.org/10.21203/rs.3.rs-26612/v1 
License: (c) (i) This work is licensed under a Creative Commons Attribution 4.0 International License. Read Full License 


\section{Abstract}

Background: In this study, we aimed to compare the efficacy and safety of removing a single-stage, retrograde, endoscopic common bile duct stone in patients with mild and moderate acute cholangitis associated with choledocholithiasis.

Methods: We enrolled 196 endoscopic retrograde cholangiopancreatography (ERCP)-naïve patients diagnosed with acute cholangitis and choledocholithiasis between September 2018 and February 2020 at a single hospital. For eligible patients, single-stage treatment involved stone removal at initial ERCP. Early ERCP was defined as ERCP performed $\leq 72$ hours following diagnosis in the emergency room.

Results: The final analysis included 138 patients. The success rate of complete stone extraction was similar in patients with mild and moderate cholangitis $(88.5 \%$ vs. $91.7 \% ; p=0.536)$. Complication rates were also comparable between the two groups. In the moderate cholangitis group, the length of hospitalization declined significantly among patients who underwent early single-stage ERCP $(10.6 \pm 6.1$ vs. $18.7 \pm 12.5$ days; $p=0.001)$ compared with patients treated with delayed ERCP. In the multivariate analysis, early ERCP indicated shorter hospitalization times ( $\leq 10$ days) (odds ratio (OR), 7.689; $p=$ 0.030 ), while endoscopic retrograde biliary drainage, for acute cholangitis only, indicated longer hospitalization times $(\mathrm{OR}, 0.358 ; p=0.030)$. A stone size larger than $1.5 \mathrm{~cm}$ was an independent risk factor for stone extraction failure (OR, 24.507; $p=0.009)$.

Conclusions: Single-stage, retrograde, endoscopic common bile duct stone removal may be safe and effective for patients with mild and moderate cholangitis. The benefit of early single-stage ERCP $(\leq 72$ hours) was reflected mainly by reduced hospitalization time and costs.

Trial registration: ClinicalTrials.gov. NCT03754491.

\section{Background}

Acute cholangitis is a medical emergency. Choledocholithiasis is the most common cause of biliary obstruction leading to cholangitis $(1,2)$. Delayed diagnosis and treatment of patients with acute cholangitis are associated with high mortality $(3,4)$. Therefore, early intervention with endoscopic retrograde cholangiopancreatography (ERCP) ( $\leq 72$ hours following diagnosis) is recommended for patients with acute cholangitis (5). In the 2013 Tokyo guidelines (6), patients with mild acute cholangitis may undergo common bile duct (CBD) stone removal and initial ERCP in patients simultaneously. However, patients with moderate acute cholangitis with choledocholithiasis require early endoscopic or percutaneous biliary drainage, or surgical biliary drainage with a T-tube. After the patient's general condition improves, CBD stones can be removed. The timing of the second ERCP for stone removal in moderate cholangitis remains variable in clinical practice. A few studies associated single-stage endoscopic treatment in mild to moderate acute cholangitis with choledocholithiasis with shorter hospitalization times. 
Further, this treatment averted the need for a second session of ERCP, thus reducing medical expenses (7-10). Therefore, the 2018 Tokyo guidelines recommend simultaneous CBD stone treatment and biliary drainage in patients with mild to moderate choledocholithiasis (11). However, evidence supporting the feasibility of single-stage stone removal in patients with moderate acute cholangitis remains insufficient. Whether or not early stone removal can reduce the bacterial load, thereby enabling better infection control and further reducing the length of hospitalization, remains unclear. Moreover, the optimal common bile stone size suitable for removal during single-stage ERCP in mild and moderate acute cholangitis is unknown. Therefore, we conducted this prospective trial to evaluate the efficacy and safety of singlestage, retrograde, endoscopic CBD stone removal in patients with mild and moderate acute cholangitis associated with choledocholithiasis.

\section{Methods}

\section{Patients and assessments}

We enrolled 196 ERCP-naïve patients diagnosed with acute cholangitis and choledocholithiasis between September 2018 and February 2020 at Kaohsiung Chang Gung Memorial Hospital, Taiwan. The institutional review board and the ethics committee of Chang Gung Memorial Hospital, Taiwan, approved this study (201701050A3), which was registered at ClinicalTrials.gov (NCT03754491). Eligible participants were at least 20 years old. Five experienced endoscopists, who conduct an average of 100 ERCPs per year, performed the procedures. The study exclusion criteria included current use of antiplatelet agents $(n=3)$, severe cholangitis $(n=8)$, inadequate sedation $(n=14)$, biliary sludge $(n=26)$, papilla not found $(n=4)$, and tumor-related obstruction $(n=3)$. We gathered written informed consent from all included patients before the trial. Before ERCP, we recorded the following demographic and clinical variables: age, sex, history of coexisting comorbidities, alcohol consumption, smoking habits, American Society of Anesthesiologists (ASA) score, and serum levels of albumin, C-reactive protein (CRP), total bilirubin, prothrombin time (PT), activated partial thromboplastin time (APTT), and liver function enzymes, as well as a complete and differential blood counts obtained in the emergency room (ER) before and after ERCP for evaluation of complications. Additionally, endoscopic findings were recorded, including papilla type (12), juxtapapillary diverticulum, CBD stone size and number, and procedure methods used.

As part of the single-stage treatment, we performed stone removal during the initial ERCP session. Early ERCP was defined as ERCP occurring 72 hours or less following diagnosis in the ER (5). Primary outcomes were as follows: 1) operation time during ERCP, defined as the period ranging from the beginning of cannulation to complete stone removal; 2) success rate of complete bile duct stone removal, 3) significant complications, including post-ERCP pancreatitis (amylase levels higher than three times the upper reference limit accompanied by abdominal pain), perforation, and bleeding; 4) length of hospitalization, and 5) costs. Secondary outcomes included the development of pneumonia within 30 days and mortality within 30 days after ERCP. Bleeding complications could present as melena or hematemesis, with a decrease in hemoglobin concentration of at least $2 \mathrm{~g} / \mathrm{dL}$. The definition of bleeding 
degree for patients who did not require transfusion was 'mild bleeding degree.' Cases requiring up to four units of blood were defined as 'moderate bleeding degree,' and those requiring five or more units of blood for transfusion, surgery, or angiography were defined as 'severe bleeding degree' (13).

\section{Stages of acute cholangitis}

The stage classification of acute cholangitis was based on the 2013 and 2018 Tokyo guidelines $(6,11)$, with a sensitivity of $91.8 \%$ and a specificity of $77.7 \%$ (14). Grade II (moderate) acute cholangitis was that associated with any two of the following conditions: 1) abnormal white blood count (WBC) count (> $12,000 / \mathrm{mm}^{3}$ or $\left.<4,000 / \mathrm{mm}^{3}\right), 2$ ) high fever $\left(\geq 39^{\circ} \mathrm{C}\right), 3$ ) age ( $\geq 75$ years), 4) hyperbilirubinemia (total bilirubin $\geq 5 \mathrm{mg} / \mathrm{dL}$ ), and 5) hypoalbuminemia ( $\angle \mathrm{SD} \times 0.7$, where standard deviation (SD) is the lower limit of the standard value). Patients with Grade III (severe) acute cholangitis exhibited dysfunction of at least one of the following organs/systems: 1) cardiovascular system, suggested by hypotension, requiring a dopamine infusion of $5 \mu \mathrm{g} / \mathrm{kg} / \mathrm{min}$ or higher or any dose of norepinephrine; 2) nervous system, indicated by a disturbance in consciousness; 3 ) respiratory system, with a $\mathrm{PaO}_{2} / \mathrm{FiO}_{2}$ ratio $>300$; 4) renal system, with oliguria and an s-creatinine level $>2 \mathrm{mg} / \mathrm{dL}$; 5 ) hepatic system, with a platelet international normalized ratio $>1.5$; and 6) hematological system, with a platelet count $<100,000 / \mathrm{mm}^{3}$. Finally, Grade I acute cholangitis was defined as acute cholangitis, not fulfilling the criteria of either Grade III (severe) or Grade II (moderate) acute cholangitis at the time of the initial diagnosis.

\section{ERCP procedure}

A side-view endoscope (JF-260V and TJF-240, Olympus Corp., Tokyo, Japan), a cholangiography catheter (PR-113Q, Olympus Corp., Tokyo, Japan), and a 0.035-in. Guidewire (Zebra exchange guidewire; Boston Scientific, Natick, MA, USA) were used in the ERCP procedures. A balloon-tipped catheter ([length, $5.5 \mathrm{~cm}$; width, 8-20 mm] Boston Scientific, Natick, MA, USA) was performed to extend papilla orifice dilatation. The balloon was inflated with saline solution to reach 8 to $20 \mathrm{~mm}$ diameter and to dilate the papilla with a progressive increase in pressure from 3 to 8 atmospheres for three minutes depending on CBD stone size (15). Endoscopic sphincterotomy (EST) was performed using standard pull-sphincterotomes (ENDOFLEX GmbH, Voerde, Germany). In cases where biliary cannulation was difficult, we performed limited precut EST or fistulotomy (Needle Knife, pointed type, ENDO-FLEX GmbH, Voerde, Germany) or a transpancreatic EST (16). A pancreas duct stent was placed for preventing post-ERCP pancreatitis (PEP) if the pancreas duct cannulation occurred twice or more. At the same time, $100 \mathrm{mg}$ of indomethacin was administered anally to all patients who did not have a history of allergy (17). Aggressive intravenous hydration (including $3 \mathrm{~mL} / \mathrm{kg} / \mathrm{h}$ during ERCP, a $20-\mathrm{mL} / \mathrm{kg}$ bolus, and $3 \mathrm{~mL} / \mathrm{kg} / \mathrm{h}$ for eight hours after ERCP) with lactated Ringer's solution was administered to all patients without contraindications (18). CBD stones were extracted using a balloon and/or basket catheter. A retrograde biliary drain with a plastic stent was inserted if $\mathrm{CBD}$ stone extraction could not be performed within one hour of the procedure, if the contrast medium bile flow was poor with papilla swelling after stone extraction, or if pus bile was noted. All patients underwent empiric antimicrobial treatment for acute cholangitis. All patients were asked to fast for at least 12 hours after ERCP and received intravenous proton-pump inhibitors (PPIs), corresponding to two doses after ERCP, and oral PPIs once daily for seven days. 


\section{Statistical analysis}

Descriptive statistics, including distributions, absolute frequencies, relative frequencies, medians with ranges, and means \pm standard deviations (SDs) were calculated depending on the variable type. Betweengroup differences for quantitative variables with normal distribution were compared using Student's $t$ test. The differences between categorical data proportions were evaluated with Fisher's exact test when there were fewer than five expected cases; otherwise, we used the chi-square test. We included factors with probability $(p)$ values $<0.3$ in the univariate analysis in the logistic regression analysis. A multivariate logistic regression model was adopted to identify independent factors of procedural success and major adverse events. A $p$ value $<0.05$ was considered to indicate statistical significance in all analyses.

\section{Results}

Population characteristics

The study included 138 patients who fulfilled the inclusion criteria, including 78 and 60 patients in the mild and moderate cholangitis groups, respectively (Fig. 1). The were no differences in sex, personal habits (i.e., alcohol use and smoking), white and platelet blood counts, APTT, levels of alanine transaminase, albumin, bilirubin, and alkaline phosphatase; estimated glomerular filtration rate (eGFR), papilla type, ratio of the juxtapapillary diverticulum, endoscopic papillary balloon dilation (EPBD), and EST between the two groups. Meanwhile, age, comorbidity rate (i.e., diabetes and hypertension), ASA score, initial body temperature $\left({ }^{\circ} \mathrm{C}\right)$ in the ER, PT, and CRP were higher in the moderate cholangitis group than in the mild cholangitis group (Table 1$)$. Stone size $(0.96 \pm 0.45 \mathrm{vs} .1 .17 \pm 0.55 \mathrm{~cm} ; p=0.128)$, mean CBD diameter ( $1.31 \pm 0.40 \mathrm{vs} .1 .50 \pm 0.39 \mathrm{~cm} ; p=0.897)$, frequency of endoscopic retrograde biliary drainage (ERBD) use $(20.5 \%$ vs. $33.3 \% ; p=0.089)$, and procedure time $(24.3 \pm 11.1$ vs. $24.8 \pm 11.3 \mathrm{~min} ; p=$ $0.929)$ did not differ between the two groups. The number of stones was higher in the mild cholangitis group than in the moderate group $(1.81 \pm 1.22 v s .1 .37 \pm 1.00$ stones; $p=0.012)$ (Table 2$)$. 
Table 1

The demographic data for the two groups (mild and moderate acute cholangitis)

\begin{tabular}{|c|c|c|c|}
\hline Characteristics & $\begin{array}{l}\text { Mild acute cholangitis } \\
(n=78, \%)\end{array}$ & $\begin{array}{l}\text { Moderate acute cholangitis } \\
(n=60, \%)\end{array}$ & $p$ value \\
\hline Age (year) (mean $\pm S D)$ & $62.8 \pm 15.8$ & $75.3 \pm 10.9$ & 0.003 \\
\hline Gender (F) & $35(44.9)$ & $23(38.3)$ & 0.395 \\
\hline Smoking & 15 (19.2) & $10(16.7)$ & 0.698 \\
\hline Alcohol & $14(17.9)$ & $9(15.0)$ & 0.645 \\
\hline Diabetes Mellitus & $14(17.9)$ & $25(41.7)$ & 0.002 \\
\hline Hypertension & $38(48.7)$ & $43(71.7)$ & 0.007 \\
\hline ASA score I/II/II/IV & $33 / 33 / 8 / 4$ & $10 / 26 / 17 / 7$ & 0.002 \\
\hline Body temperature $\left({ }^{\circ} \mathrm{C}\right)$ & $36.8 \pm 0.9$ & $37.9 \pm 1.4$ & $<0.001$ \\
\hline WBC $(\times 1000 / \mu L)$ & $10.2 \pm 5.5$ & $14.2 \pm 4.3$ & 0.116 \\
\hline Platelet $(\times 1000 / \mu \mathrm{L})$ & $238.2 \pm 91.8$ & $202.2 \pm 90.1$ & 0.479 \\
\hline PT (s) & $10.7 \pm 0.8$ & $11.7 \pm 2.6$ & 0.016 \\
\hline APTT (s) & $27.6 \pm 3.5$ & $29.9 \pm 4.6$ & 0.057 \\
\hline Albumin (mg/dL) & $3.7 \pm 0.5$ & $3.3 \pm 0.4$ & 0.801 \\
\hline eGFR (mL/min/1.73²) & $70.5 \pm 33.8$ & $49.3 \pm 24.7$ & 0.071 \\
\hline AST (U/L) & $199.2 \pm 246.1$ & $219.4 \pm 186.8$ & 0.877 \\
\hline ALT (U/L) & $254.7 \pm 206.4$ & $243.9 \pm 200.1$ & 0.568 \\
\hline Bilirubin (total) (mg/dL) & $5.8 \pm 4.7$ & $6.2 \pm 4.1$ & 0.128 \\
\hline ALK-P (U/L) & $208.1 \pm 134.8$ & $245.9 \pm 165.7$ & 0.377 \\
\hline CRP (U/L) & $47.7 \pm 68.3$ & $122.0 \pm 80.9$ & 0.017 \\
\hline \multicolumn{4}{|c|}{$\begin{array}{l}\text { Abbreviations: ASA, American Society of Anesthesiology score; WBC, white blood cell; PT, prothrombin } \\
\text { time; APTT, activated partial thromboplastin time; eGFR, estimated glomerular filtration rate; AST, } \\
\text { aspartate aminotransferase; ALT, alanine aminotransferase; ALK-P, Alkaline phosphatase; CRP, C- } \\
\text { reactive protein. }\end{array}$} \\
\hline
\end{tabular}


Table 2

Endoscopic findings and outcomes in patients with mild and moderate acute cholangitis.

\begin{tabular}{|llll|}
\hline Characteristics & $\begin{array}{l}\text { Mild acute cholangitis } \\
(\mathbf{n}=\mathbf{7 8 ,} \%)\end{array}$ & $\begin{array}{l}\text { Moderate acute cholangitis } \\
(\mathbf{n}=\mathbf{6 0}, \boldsymbol{)})\end{array}$ & $\boldsymbol{p}$ value \\
\hline Pancreas duct filling & $2(2.7)$ & $4(6.8)$ & 0.260 \\
\hline Papilla type(I IV) & $61 / 8 / 5 / 4$ & $47 / 8 / 3 / 2$ & 0.886 \\
\hline Periampullary diverticulum & $28(36.4)$ & $30(50.0)$ & 0.096 \\
\hline EPBD & $67(93.1)$ & $52(89.7)$ & 0.489 \\
\hline EST & $17(21.8)$ & $10(16.7)$ & 0.452 \\
\hline Stones size (cm) & $0.96 \pm 0.45$ & $1.17 \pm 0.55$ & 0.128 \\
\hline Stone number & $1.81 \pm 1.22$ & $1.37 \pm 1.00$ & 0.012 \\
\hline Mean CBD diameter (cm ) & $1.31 \pm 0.40$ & $1.50 \pm 0.39$ & 0.897 \\
\hline Balloon extraction & $68(94.4)$ & $54(93.1)$ & 0.752 \\
\hline Basket use & $9(12.5)$ & $11(19.0)$ & 0.310 \\
\hline Complete stone removal & $69(88.5)$ & $55(91.7)$ & 0.536 \\
\hline ERBD & $16(20.5)$ & $20(33.3)$ & 0.089 \\
\hline Procedure Time (min) & $24.3 \pm 11.1$ & $24.8 \pm 11.3$ & 0.929 \\
\hline Hospital stay (days) & $11.9 \pm 6.2$ & $14.3 \pm 10.2$ & 0.014 \\
\hline Hospital cost (USD) & $3005.7 \pm 1302.7$ & $4011.7 \pm 3048.8$ & $<0.001$ \\
\hline Abbreviations: EPBD, endoscopic papillary balloon dilation; EST, endoscopic sphincterotomy; CBD, \\
\hline common bile duct; ERBD, Endoscopic retrograde biliary drainage
\end{tabular}

Treatment outcomes and complications

The complete stone extraction success rate was similar in the mild and moderate cholangitis groups (88.5\% vs. $91.7 \% ; p=0.536)$. Additionally, the complication rates were comparable between the two groups (PEP, $5.1 \%$ vs. $1.7 \%, p=0.281$; bleeding, $1.3 \%$ vs. $0 \%, p=0.379$; perforation, $1.3 \%$ vs. $0 \%, p=0.379$; pneumonia, $1.3 \%$ vs. $1.7 \%, p=0.851)$. However, the moderate cholangitis group experienced longer hospitalization ( $14.3 \pm 10.2$ vs. $11.9 \pm 6.2$ days; $p=0.014)$ and higher costs $(4011.7 \pm 3048.8 v s$. $3005.7 \pm$ 1302.7 USD; $p<0.001$ ), as shown in Table 3. There were no fatalities in any of the two groups. One patient experienced coffee-ground vomiting and a mild degree of bleeding after EPBD. Five patients with a mild degree of PEP were treated with conservative care, with symptoms spontaneously resolving. 
Table 3

Adverse events observed after ERCP in patients with mild and moderate acute cholangitis

\begin{tabular}{|llll|}
\hline Adverse events & $\begin{array}{l}\text { Mild cholangitis } \\
(\mathbf{n}=\mathbf{7 8}, \boldsymbol{\%})\end{array}$ & $\begin{array}{l}\text { Moderate cholangitis } \\
(\mathbf{n}=\mathbf{6 0}, \%)\end{array}$ & $\boldsymbol{p}$ value \\
\hline PEP & $4(5.1)$ & $1(1.7)$ & 0.281 \\
\hline Bleeding & $1(1.3)$ & $0(0)$ & 0.379 \\
\hline Perforation & $1(1.3)$ & $0(0)$ & 0.379 \\
\hline Pneumonia (30 days) & $1(1.3)$ & $1(1.7)$ & 0.851 \\
\hline Mortality (30 days) & $0(0)$ & $0(0)$ & - \\
\hline Abbreviations: ERCP, endoscopic retrograde cholangiopancreatography; PEP, post-ERCP pancreatitis.
\end{tabular}

Timing of single-stage stone extraction during ERCP

In the subgroup analysis of early ERCP (mean \pm SD, $36.1 \pm 20.9 \mathrm{~h}$ ), there were similar rates of successful complete stone extraction and ERCP complications in the two groups. We performed ERBD more often for the moderate cholangitis group than the mild group $(45.5 \%$ vs. $24.5 \% ; p=0.044)$. However, both groups had similar hospitalization lengths and costs. The subgroup analysis of delayed ERCP (mean \pm SD, 176.5 $\pm 114.8 \mathrm{~h}$ ) indicated similar success rates of complete stone extraction and ERCP complications in the two groups. The patients in the moderate cholangitis group who were treated with initial PTBD $(37.0 \% \mathrm{vs}$. $8.0 \% ; p=0.013)$ had longer hospital stays $(18.7 \pm 12.5 \mathrm{vs}$. $15.7 \pm 6.8$ days; $p=0.016)$ and higher costs $(4967.3 \pm 3806.8$ vs. $3290.7 \pm 1180.7$ USD; $p=0.001)$ than the mild cholangitis group (Table 4). Among patients treated with early ERCP, the length of hospitalization declined significantly in the moderate cholangitis group $(10.6 \pm 6.1$ vs. $18.7 \pm 12.5$ days; $p=0.001)$. There was also a declining trend in the mild cholangitis group ( $10.0 \pm 5.0$ vs. $15.7 \pm 6.8$ days; $p=0.056)$ relative to the group treated with delayed ERCP (Fig. 2). In the univariate and multivariate analyses of the factors associated with shorter hospital stay ( $\leq 10$ days), early ERCP indicated reduced hospitalization (odds ratio [OR], 7.689, 95\% confidence interval [Cl], 3.168-18.657; $p=0.030)$. Meanwhile, the presence of liver cirrhosis $(\mathrm{OR}, 0.101,95 \% \mathrm{Cl}$, $0.011-0.904 ; p=0.040)$ and ERBD treatment $(\mathrm{OR}, 0.358,95 \% \mathrm{Cl}, 0.142-0.904 ; p=0.030)$ were factors suggesting longer hospitalization (Table 5). 
Table 4

Clinical characteristics and outcomes according to ERCP timing according to type of cholangitis (mild $v s$. moderate).

\begin{tabular}{|c|c|c|c|c|c|c|}
\hline \multirow{2}{*}{$\begin{array}{l}\text { ERCP timing } \\
\text { from ER } \\
\text { (Mean } \pm \text { SD) } \\
\text { (hrs) }\end{array}$} & \multicolumn{3}{|c|}{ Early ( $\leq 72 \mathrm{hrs})(36.1 \pm 20.9)$} & \multicolumn{3}{|c|}{ Late $(>72 \mathrm{hrs})(176.5 \pm 114.8)$} \\
\hline & $\begin{array}{l}\text { Mild } \\
\text { cholangitis } \\
(n=53, \%)\end{array}$ & $\begin{array}{l}\text { Moderate } \\
\text { cholangitis } \\
(n=33, \%)\end{array}$ & $\begin{array}{l}p \\
\text { value }\end{array}$ & $\begin{array}{l}\text { Mild } \\
\text { cholangitis } \\
(n=25, \%)\end{array}$ & $\begin{array}{l}\text { Moderate } \\
\text { cholangitis } \\
(n=27, \%)\end{array}$ & $\begin{array}{l}p \\
\text { value }\end{array}$ \\
\hline Age $(y / o)$ & $62.2 \pm 16.3$ & $75.4 \pm 11.0$ & 0.001 & $64.1 \pm 14.9$ & $75.7 \pm 11.0$ & 0.014 \\
\hline CRP (U/I) & $46.6 \pm 64.5$ & $125.5 \pm 70.3$ & 0.012 & $50.0 \pm 76.9$ & $117.6 \pm 93.9$ & 0.020 \\
\hline Stone $\geq 1.5 \mathrm{~cm}$ & $4(7.5)$ & $7(21.2)$ & 0.065 & $5(20.0)$ & $7(25.9)$ & 0.612 \\
\hline $\begin{array}{l}\text { Complete CBD } \\
\text { removal }\end{array}$ & $49(92.5)$ & $31(93.9)$ & 0.792 & $20(80.0)$ & $24(88.9)$ & 0.375 \\
\hline PEP & $3(5.7)$ & $1(3.0)$ & 0.573 & $1(4.0)$ & $0(0)$ & 0.294 \\
\hline Bleeding & $1(1.9)$ & $0(0)$ & 0.427 & $0(0)$ & $0(0)$ & - \\
\hline Perforation & $0(0)$ & $0(0)$ & - & $1(4.0)$ & $0(0)$ & 0.294 \\
\hline Initial PTBD & $2(3.8)$ & $1(3.0)$ & 0.855 & $2(8.0)$ & $10(37.0)$ & 0.013 \\
\hline ERBD & $13(24.5)$ & $15(45.5)$ & 0.044 & $3(12)$ & 5 (18.5) & 0.515 \\
\hline $\begin{array}{l}\text { Hospital stay } \\
\text { (days) }\end{array}$ & $10.0 \pm 5.0$ & $10.6 \pm 6.1$ & 0.433 & $15.7 \pm .6 .8$ & $18.7 \pm 12.5$ & 0.016 \\
\hline $\begin{array}{l}\text { Hospital cost } \\
\text { (USD) }\end{array}$ & $\begin{array}{l}2877.6 \pm \\
1341.6\end{array}$ & $\begin{array}{l}3250.2 \pm \\
2036.0\end{array}$ & 0.177 & $\begin{array}{l}3290.7 \pm \\
1180.7\end{array}$ & $\begin{array}{l}4967.3 \pm \\
3806.8\end{array}$ & 0.001 \\
\hline
\end{tabular}


Table 5

Univariate and multivariate analyses of the factors associated with reduced length of hospitalization ( $\leq$ 10 days)

\begin{tabular}{|c|c|c|c|c|c|}
\hline Variants & Comparison & $\begin{array}{l}\text { Univariate } \\
\text { OR (95\% C.I.) }\end{array}$ & $\begin{array}{l}p \\
\text { value }\end{array}$ & $\begin{array}{l}\text { Multivariate } \\
\text { OR (95\% C.I.) }\end{array}$ & $\begin{array}{l}p \\
\text { value }\end{array}$ \\
\hline \multicolumn{6}{|l|}{ Clinical factors } \\
\hline Age $\geq 75$ y/o & $\begin{array}{l}\text { Yes versus } \\
\text { no }\end{array}$ & $\begin{array}{l}0.689(0.352- \\
1.343)\end{array}$ & 0.272 & $\begin{array}{l}1.663(0.500- \\
5.538)\end{array}$ & 0.407 \\
\hline Gender & $\begin{array}{l}\text { Male versus } \\
\text { female }\end{array}$ & $\begin{array}{l}0.976(0.497- \\
1.915)\end{array}$ & 0.943 & & \\
\hline $\mathrm{BMI} \geq 24$ & $\begin{array}{l}\text { Yes versus } \\
\text { no }\end{array}$ & $\begin{array}{l}1.016(0.476- \\
2.169)\end{array}$ & 0.967 & & \\
\hline Diabetes & $\begin{array}{l}\text { Yes versus } \\
\text { no }\end{array}$ & $\begin{array}{l}0.593(0.280- \\
1.254)\end{array}$ & 0.171 & $\begin{array}{l}0.683(0.219- \\
2.124)\end{array}$ & 0.510 \\
\hline Hypertension & $\begin{array}{l}\text { Yes versus } \\
\text { no }\end{array}$ & $\begin{array}{l}0.565(0.286- \\
1.117)\end{array}$ & 0.101 & $\begin{array}{l}0.622(0.219- \\
1.766)\end{array}$ & 0.372 \\
\hline $\begin{array}{l}\text { Cerebrovascular } \\
\text { accident }\end{array}$ & $\begin{array}{l}\text { Yes versus } \\
\text { no }\end{array}$ & $\begin{array}{l}0.470(0.113- \\
1.961)\end{array}$ & 0.300 & $\begin{array}{l}0.761(0.102- \\
5.694)\end{array}$ & 0.791 \\
\hline $\begin{array}{l}\text { Coronary artery } \\
\text { disease }\end{array}$ & $\begin{array}{l}\text { Yes versus } \\
\text { no }\end{array}$ & $\begin{array}{l}0.528(0.228- \\
1.222)\end{array}$ & 0.136 & $\begin{array}{l}0.833(0.228- \\
3.052)\end{array}$ & 0.783 \\
\hline Liver cirrhosis & $\begin{array}{l}\text { Yes versus } \\
\text { no }\end{array}$ & $\begin{array}{l}0.109(0.013- \\
0.894)\end{array}$ & 0.039 & $\begin{array}{l}0.101(0.011- \\
0.904)\end{array}$ & 0.040 \\
\hline $\begin{array}{l}\text { Chronic kidney } \\
\text { disease } \\
(\text { eGFR< } \\
60 \mathrm{ml} / \mathrm{min} / 1.73^{2} \text { ) }\end{array}$ & $\begin{array}{l}\text { Yes versus } \\
\text { no }\end{array}$ & $\begin{array}{l}0.568(0.282- \\
1.143)\end{array}$ & 0.113 & $\begin{array}{l}0.483(0.140- \\
1.665)\end{array}$ & 0.249 \\
\hline Stage of cholangitis & $\begin{array}{l}\text { Moderate } \\
\text { versus Mild }\end{array}$ & $\begin{array}{l}0.727(0.371- \\
1.424)\end{array}$ & 0.353 & & \\
\hline Initial PTBD & $\begin{array}{l}\text { Yes versus } \\
\text { no }\end{array}$ & $\begin{array}{l}0.116(0.025- \\
0.530)\end{array}$ & 0.006 & $\begin{array}{l}0.363(0.046- \\
2.847)\end{array}$ & 0.335 \\
\hline Biliary pancreatitis & $\begin{array}{l}\text { Yes versus } \\
\text { no }\end{array}$ & $\begin{array}{l}0.792(0.347- \\
1.809)\end{array}$ & 0.579 & & \\
\hline
\end{tabular}

Factors with $p$ values less than 0.3 were included in the logistic regression analysis. Abbreviations: $\mathrm{OR}$, odd ratio; $\mathrm{BMI}$, body mass index; PTBD, percutaneous transhepatic biliary drainage; WBC, white blood count; CRP, C-reactive protein; EST, endoscopic sphincterotomy; EPBD, endoscopic papillary balloon dilation; ERBD, Endoscopic retrograde biliary drainage; ERCP, endoscopic retrograde cholangiopancreatography. 


\begin{tabular}{|c|c|c|c|c|c|}
\hline Variants & Comparison & $\begin{array}{l}\text { Univariate } \\
\text { OR (95\% C.I.) }\end{array}$ & $\begin{array}{l}p \\
\text { value }\end{array}$ & $\begin{array}{l}\text { Multivariate } \\
\text { OR (95\% C.I.) }\end{array}$ & $\begin{array}{l}p \\
\text { value }\end{array}$ \\
\hline $\begin{array}{l}W B C \geq 12,000 \\
\text { or }<4,000(\times 1000 / \mu \mathrm{L})\end{array}$ & $\begin{array}{l}\text { Yes versus } \\
\text { no }\end{array}$ & $\begin{array}{l}0.576(0.294- \\
1.128)\end{array}$ & 0.108 & $\begin{array}{l}1.520(0.541- \\
4.273)\end{array}$ & 0.427 \\
\hline $\mathrm{CRP} \geq 100(\mathrm{U} / \mathrm{I})$ & $\begin{array}{l}\text { Yes versus } \\
\text { no }\end{array}$ & $\begin{array}{l}0.583(0.287- \\
1.186)\end{array}$ & 0.136 & $\begin{array}{l}0.736(0.261- \\
2.078)\end{array}$ & 0.563 \\
\hline Bilirubin $\geq 5(\mathrm{mg} / \mathrm{dL})$ & $\begin{array}{l}\text { Yes versus } \\
\text { no }\end{array}$ & $\begin{array}{l}0.749(0.383- \\
1.462)\end{array}$ & 0.396 & & \\
\hline \multicolumn{6}{|l|}{ Endoscopic factors } \\
\hline Stone $\geq 1.5 \mathrm{~cm}$ & $\begin{array}{l}\text { Yes versus } \\
\text { no }\end{array}$ & $\begin{array}{l}0.708(0.286- \\
1.738)\end{array}$ & 0.448 & & \\
\hline EST & $\begin{array}{l}\text { Yes versus } \\
\text { no }\end{array}$ & $\begin{array}{l}0.418(0.173- \\
1.010)\end{array}$ & 0.053 & $\begin{array}{l}0.355(0.111- \\
1.142)\end{array}$ & 0.082 \\
\hline EPBD & $\begin{array}{l}\text { Yes versus } \\
\text { no }\end{array}$ & $\begin{array}{l}2.407(0.687- \\
8.431)\end{array}$ & 0.169 & $\begin{array}{l}0.966(0.193- \\
4.833)\end{array}$ & 0.967 \\
\hline ERBD & $\begin{array}{l}\text { Yes versus } \\
\text { no }\end{array}$ & $\begin{array}{l}0.601(0.283- \\
1.316)\end{array}$ & 0.208 & $\begin{array}{l}0.358(0.142- \\
0.904)\end{array}$ & 0.030 \\
\hline ERCP timing $\leq 72 \mathrm{hrs}$ & $\begin{array}{l}\text { Yes versus } \\
\text { no }\end{array}$ & $\begin{array}{l}5.067(2.378- \\
10.795)\end{array}$ & $<.001$ & $\begin{array}{l}7.689(3.168- \\
18.657)\end{array}$ & $\begin{array}{l}< \\
0.001\end{array}$ \\
\hline \multicolumn{6}{|c|}{$\begin{array}{l}\text { Factors with } p \text { values less than } 0.3 \text { were included in the logistic regression analysis. Abbreviations: } \\
\text { OR, odd ratio; BMI, body mass index; PTBD, percutaneous transhepatic biliary drainage; WBC, white } \\
\text { blood count; CRP, C-reactive protein; EST, endoscopic sphincterotomy; EPBD, endoscopic papillary } \\
\text { balloon dilation; ERBD, Endoscopic retrograde biliary drainage; ERCP, endoscopic retrograde } \\
\text { cholangiopancreatography. }\end{array}$} \\
\hline
\end{tabular}

Stone size during single-stage stone extraction by ERCP

In 14 cases, we were unable to extract stones. Eight cases were due to difficult cannulation, even with precut EST or fistulotomy use, while six cases were a simple failure of stone extraction. We conducted univariate and multivariate analyses of the factors associated with CBD stone extraction failure. After excluding cannulation failure cases, stone sizes greater than $1.5 \mathrm{~cm}$ was an independent factor suggesting $\mathrm{CBD}$ stone extraction failure $(\mathrm{OR}, 24.507 ; 95 \% \mathrm{Cl}, 2.186-274.708 ; p=0.009)$ (Table 6). In the subgroup analysis, the rate of complete CBD stone removal varied according to CBD stone size (i.e., $\leq$ $1.5 \mathrm{~cm}$ or $>1.5 \mathrm{~cm}$ ). Thus, after excluding cannulation failure cases, there were higher success rates ( $98.6 \%$ and $100 \%$, respectively) among patients with CBD stones $\leq 1.5 \mathrm{~cm}$ than there were among patients with stones $>1.5 \mathrm{~cm}(81.8 \%$ and $70.0 \%$, respectively), regardless of whether ERCP was performed early or not ( $p=0.005$ and $p=0.001$, respectively) (Fig. 3). 
Table 6

Univariate and multivariate analyses of the factors associated with failure of the CBD stone extraction (excluding cases with cannulation failure)

\begin{tabular}{|c|c|c|c|c|c|}
\hline Variants & Comparison & $\begin{array}{l}\text { Univariate } \\
\text { OR (95\% C.I.) }\end{array}$ & $\begin{array}{l}p \\
\text { value }\end{array}$ & $\begin{array}{l}\text { Multivariate } \\
\text { OR (95\% C.I.) }\end{array}$ & $\begin{array}{l}p \\
\text { value }\end{array}$ \\
\hline Age $\geq 75$ y/o & $\begin{array}{l}\text { Yes versus } \\
\text { no }\end{array}$ & $\begin{array}{l}6.481(0.735- \\
57.119)\end{array}$ & 0.092 & $\begin{array}{l}1.997(0.133- \\
29.886)\end{array}$ & 0.616 \\
\hline $\begin{array}{l}\text { Stage of } \\
\text { cholangitis }\end{array}$ & $\begin{array}{l}\text { Moderate } \\
\text { versus Mild }\end{array}$ & $1.255(0.244-6.461)$ & 0.786 & & \\
\hline$C R P \geq 100$ & $\begin{array}{l}\text { Yes versus } \\
\text { no }\end{array}$ & $\begin{array}{l}3.951(0.695- \\
22.477)\end{array}$ & 0.121 & $\begin{array}{l}1.958(0.217- \\
17.631)\end{array}$ & 0.549 \\
\hline $\begin{array}{l}\text { ERCP timing } \leq \\
72 \mathrm{hrs}\end{array}$ & $\begin{array}{l}\text { Yes versus } \\
\text { no }\end{array}$ & $0.550(0.106-2.841)$ & 0.475 & & \\
\hline Diverticulum & $\begin{array}{l}\text { Yes versus } \\
\text { no }\end{array}$ & $0.259(0.029-2.285)$ & 0.224 & $0.230(0.021-2.476)$ & 0.225 \\
\hline Stone $>1.5 \mathrm{~cm}$ & $\begin{array}{l}\text { Yes versus } \\
\text { no }\end{array}$ & $\begin{array}{l}33.750(3.701- \\
307.747)\end{array}$ & 0.002 & $\begin{array}{l}24.507(2.186- \\
274.708)\end{array}$ & 0.009 \\
\hline $\begin{array}{l}\text { Distal CBD } \\
\text { narrowing }\end{array}$ & $\begin{array}{l}\text { Yes versus } \\
\text { no }\end{array}$ & $\begin{array}{l}1.796(0.312- \\
10.338)\end{array}$ & 0.512 & & \\
\hline EST & $\begin{array}{l}\text { Yes versus } \\
\text { no }\end{array}$ & $\begin{array}{l}4.905(0.925- \\
25.995)\end{array}$ & 0.062 & $\begin{array}{l}3.230(0.393- \\
26.520)\end{array}$ & 0.275 \\
\hline EPBD & $\begin{array}{l}\text { Yes versus } \\
\text { no }\end{array}$ & $0.439(0.047-4.129)$ & 0.471 & & \\
\hline $\begin{array}{l}\text { Factors with } p \text { v } \\
\text { CBD, common b } \\
\text { cholangiopancr } \\
\text { dilation. }\end{array}$ & $\begin{array}{l}\text { less than } 0.3 \\
\text { ct; OR, odd rat } \\
\text { raphy; EST, en }\end{array}$ & $\begin{array}{l}\text { ere included in the logi } \\
\text {; CRP, C-reactive protei } \\
\text { scopic sphincterotom }\end{array}$ & $\begin{array}{l}\text { Ic regres } \\
\text { ERCP, er } \\
\text { EPBD, e }\end{array}$ & $\begin{array}{l}\text { ion analysis. Abbreviat } \\
\text { loscopic retrograde } \\
\text { doscopic papillary ball }\end{array}$ & \\
\hline
\end{tabular}

\section{Discussion}

To our knowledge, this is the first prospective trial that aimed to identify suitable ERCP timing and the distribution of CBD stone sizes, as observed during single-stage, retrograde, endoscopic stone removal in patients with acute cholangitis and to compare differences between the mild and moderate stages of acute cholangitis that may affect treatment strategy. The most worrying issue under review in this investigation was whether direct stone extraction in mild to moderate acute cholangitis would increase complications, such as bleeding and pancreatitis. The institutional review board and ethics committee recommended that we adopt standard prevention strategies to reduce injuries, such as guidewire-based selective cannulation, long-term dilatation during EPBD, pancreas duct stenting, indomethacin use, aggressive hydration, and PPI administration. 
Endoscopists skilled in ERCP can clear CBD stones successfully after EST in the early stages of mild cholangitis. However, adding EST may introduce a higher risk of unforeseen complications, such as bleeding $(4-14.5 \%)(19,20)$. Acute cholangitis seems to be an independent risk factor for post-EST bleeding $(14,21)$. Therefore, biliary drainage without sphincterotomy is recommended in patients with severe acute cholangitis (19). In a national, population-based study by Hung et al. (22), EPBD was the preferred method to decrease the risk of post-ERCP hemorrhage, especially in patients with liver cirrhosis or impaired renal function. Thus, we typically opt for EPBD ( 90\%) for stone removal in cases of acute cholangitis to reduce bleeding events. In this study, there was no case of post-EST bleeding (0/27; $0 \%)$, not even among the seven cases with transbiliary EST, eleven cases with fistulotomy, seven cases with limited precut EST, or the patient who received transpancreatic EST. One patient did experience mild bleeding after EPBD. The bleeding risk of EST in mild and moderate cholangitis may, therefore, be minimal and acceptable. Similarly, Ito et al. (7) associated no complications, such as pancreatitis, bleeding, or perforation, with procedures performed by experienced specialists concerning immediate EST for acute suppurative cholangitis. In our study cohort, there was an acceptable PEP complication rate $(5 / 138 ; 3.6 \%)$ with the use of standard prevention strategies.

In clinical practice, the optimal timing for stone removal in moderate cholangitis varies. For example, Hui et al. (23) performed a second ERCP procedure on all patients four to eight weeks after the first operation for bile duct stone removal. In the study by Ito et al. (7), all patients underwent a second elective EST procedure one week after the first intervention for bile duct stone removal. Eto et al. (8) reported a $90 \%$ success rate for stone removal after single-stage treatment in patients with mild to moderate acute cholangitis, with an acceptable bleeding rate of $4 \%(2 / 50)$. However, these authors did not comment in detail on the differences in ERCP timing. In our study, among patients with acute cholangitis who were treated with early single-stage ERCP, hospitalization length declined significantly in the moderate cholangitis group (10.6 \pm 6.1 vs. $18.7 \pm 12.5$ days; $p=0.001)$ and somewhat in the mild cholangitis group (10.0 \pm 5.0 vs. $15.7 \pm 6.8$ days; $p=0.056)$ compared with those treated with delayed ERCP (Fig. 2$)$. In the multivariate analysis, early ERCP was an independent factor predicting shorter hospitalization (OR, 7.689; $p=0.030)$.

Meanwhile, initial PTBD for acute cholangitis did not reduce the length of hospitalization. We suspect that patients with moderate cholangitis who receive early or delayed ERCP have different inflammation severity levels. In the sub-analysis of baseline characteristics of patients with moderate acute cholangitis who underwent early and delayed ERCP, respectively, there was no difference between the two groups in terms of age, renal function, albumin, WBC count, liver function, or bilirubin. However, patients with moderate cholangitis, who underwent early ERCP relative to delayed ERCP (125.5 \pm 70.3 vs. 117.6 \pm 93.9; $p=0.029)$, experienced a higher level of CRP. Therefore, it appears reasonable to suggest that the optimal timing of single-stage stone removal in both mild and moderate cholangitis is within $72 \mathrm{~h}$.

On the other hand, there was a negative association between biliary drainage only (ERBD) for acute cholangitis and successful infection control as well as reduced hospital stay $(\mathrm{OR}: 0.358, p=0.030)$ (Table 5). As shown in other studies addressing acute cholecystitis management, an early approach to 
emergency cholecystectomy within $72 \mathrm{~h}$ of symptom onset reduces operative time, decreases hospitalization length, is associated with fewer adverse postoperative outcomes, and reduces mortality (24-26). The circumstance might be related to the management of foreign-body infection (27): Removing debris from the site of injury reduces the bacterial load and thereby facilitates control infection. Bactibilia (the presence of bacteria in the biliary tract) increases in the presence of biliary obstruction, mainly partial obstruction, and in the presence of foreign bodies like stones (28). The most common bacteria linked to ascending cholangitis are Escherichia coli (29), Klebsiella (30), Enterobacter (31), and Enterococcus (32), which form a biofilm covering the surfaces of stones. This biofilm protects the bacteria from antibacterial agents and phagocytic leucocytes (33). Therefore, prompt removal of infected stones in cases of acute cholangitis is preferable.

The evaluation of stone size during single-stage removal in acute cholangitis is important. We determined that stones that it was not challenging to remove stones up to $1.5 \mathrm{~cm}(34)$, and successful stone removal (98.6-100\%) was higher than when the stones were larger than $1.5 \mathrm{~cm}$ (success rate, $70.0-81.8 \%$ ), as long as biliary cannulation was successful (Fig. 3). Thus, we recommend direct removal of stones in patients with mild and moderate cholangitis only if the stone size is $1.5 \mathrm{~cm}$ or smaller.

The limitations of the current study need to be acknowledged. First, this study was initially designed as a randomized controlled trial to compare single-stage and two-stage ERCP-based stone removal (biliary drainage first and bile stone removal one week later) in patients with moderate acute cholangitis. However, most patients refused to undergo two-stage ERCP because of the need for more than one session. Therefore, we altered the trial design to a prospective trial of single-stage ERCP in patients with mild and moderate cholangitis. The results indicated that treatment by biliary drainage only (ERBD) in acute cholangitis (OR: $0.358, p=0.030$ ) was negatively associated with a shorter hospital stay. Second, although limited EST plus endoscopic papillary large-balloon dilation to remove large bile duct stones (> $1.5 \mathrm{~cm}$ ) was associated with a high success rate (98.3\%) in our previous study (35), a single-stage treatment for larger stones $(>1.5 \mathrm{~cm})$ in patients with moderate cholangitis might be more complicated. More research is required to assess the benefits and risks. Definitive treatment with removal of large stones is still recommended, but only after the patient's general condition becomes stable per the established guidelines.

\section{Conclusions}

We conclude that patients with moderate acute cholangitis have more comorbidity and a more severe degree of inflammation, resulting in longer hospitalization and higher hospitalization costs. Single-stage, retrograde, endoscopic $\mathrm{CBD}$ stone removal in mild and moderate cholangitis with choledocholithiasis may be safe and effective, especially if the stone is $1.5 \mathrm{~cm}$ or smaller, which can obviate the requirement for a second ERCP session, thus reducing medical expenses. In this study, we demonstrated the benefit of single-stage emergency ERCP by shorter hospital stays and reduced costs for patients with mild and moderate cholangitis. However, large-scale, randomized clinical trials are necessary to clarify the safety 
and efficacy of the single-stage approach, especially when dealing with large CBD stones larger than $1.5 \mathrm{~cm}$.

\section{List Of Abbreviations}

ERCP, endoscopic retrograde cholangiopancreatography; CBD, common bile duct; ASA, American Society of Anesthesiologists score; CRP, C-reactive protein; PT, prothrombin time; APTT, activated partial thromboplastin time; ER, emergency room; WBC, white blood count; SD, standard deviation; eGFR, estimated glomerular filtration rate; AST, aspartate aminotransferase; ALT, alanine aminotransferase; ALKP, Alkaline phosphatase; EPBD, endoscopic papillary balloon dilation; EST, endoscopic sphincterotomy; ERBD, Endoscopic retrograde biliary drainage; PEP, post-ERCP pancreatitis; PTBD, percutaneous transhepatic biliary drainage; OR, odd ratio; $\mathrm{BMI}$, body mass index.

\section{Declarations}

\section{Ethics approval and consent to participate:}

The study was approved by both the institutional review board and the ethics committee of Chang Gung Memorial Hospital, Taiwan (201701050A3) and registered at ClinicalTrials.gov (NCT03754491). All participants provided written informed consent before the trial.

\section{Consent for Publication:}

All authors will accept full responsibility for the conduct of the study. All authors have had access to the data and have control of the decision to publish.

\section{Availability of data and material:}

The authors confirm that the data supporting the findings of this study are available within the article and its supplementary materials.

\section{Competing interests:}

The author reports no conflicts of interest in this work. Co-author Seng-Kee Chuah is an Associate Editor of BMC Gastroenterology.

\section{Funding:}

This work was financially supported by the Kaohsiung Chang Gung Memorial Hospital (CMRPG8H0341), and assisted with statistical analysis. 


\section{Authors' contributions:}

CMK participated in the design of the study, coordinated the study, and performed the statistical analysis. CML wrote the manuscript. YCC, LSL, CKW, FMS, PYH, TLM, CHK, SKC contributed with administrative, technical, and/or material support. All authors have read and approved the manuscript

\section{Acknowledgments}

The authors would like to thank Miss Yu-Huei Wu in the Division of Gastroenterology, Kaohsiung Chang Gung Memorial Hospital for her assistance with programming. The authors also appreciate the Biostatistics Center, Kaohsiung Chang Gung Memorial Hospital, for assistance with statistical analyses.

\section{References}

1. Kimura Y, Takada T, Strasberg SM, Pitt HA, Gouma DJ, Garden OJ, et al. TG13 current terminology, etiology, and epidemiology of acute cholangitis and cholecystitis. J Hepatobil Pancreat Sci. 2013;20:8-23.

2. Kinney TP. Management of ascending cholangitis. Gastrointest Endosc Clin N Am. 2007;17:289306. vi.

3. Chopra KB, Peters RA, O'Toole PA, Williams SG, Gimson AE, Lombard MG, et al. Randomised study of endoscopic biliary endoprosthesis versus duct clearance for bileduct stones in high-risk patients. Lancet. 1996;348:791-3.

4. Parikh MP, Wadhwa V, Thota PN, Lopez R, Sanaka MR. Outcomes associated with timing of ERCP in acute cholangitis secondary to choledocholithiasis. J Clin Gastroenterol. 2018;52:e97-102.

5. ASGE Standards of Practice Committee. Maple JT, Ikenberry SO, Anderson MA, Appalaneni V, Decker $\mathrm{GA}$, et al. The role of endoscopy in the management of choledocholithiasis. Gastrointest Endosc. 2011;74:731-44.

6. Miura F, Takada T, Strasberg SM, Solomkin JS, Pitt HA, Gouma DJ, et al. TG13 flowchart for the management of acute cholangitis and cholecystitis. J Hepatobil Pancreat Sci. 2013;20:47-54.

7. Ito T, Sai JK, Okubo H, Saito H, Ishii S, Kanazawa R, et al. Safety of immediate endoscopic sphincterotomy in acute suppurative cholangitis caused by choledocholithiasis. World J Gastrointest Endosc. 2016;8:180-5.

8. Eto K, Kawakami H, Haba S, Yamato H, Okuda T, Yane K, et al. Single-stage endoscopic treatment for mild to moderate acute cholangitis associated with choledocholithiasis: a multicenter, nonrandomized, open-label and exploratory clinical trial. J Hepatobil Pancreat Sci. 2015;22:825-30.

9. Jang SE, Park SW, Lee BS, Shin CM, Lee SH, Kim JW, et al. Management for CBD stone-related mild to moderate acute cholangitis: urgent versus elective ERCP. Dig Dis Sci. 2013;58:2082-7. 
10. Hung KT, Kuo CM, Tsai CE, Chiu Y, Lu L, Wu C, et al. Single-stage retrograde endoscopic common bile duct stone removal might be sufficient in moderate acute cholangitis with a stone size $\leq 12 \mathrm{~mm}$ : $\mathrm{A}$ retrospective cohort study with propensity score matching. Adv Dig Med. 2020:1-7.

11. Miura F, Okamoto K, Takada T, Strasberg SM, Asbun HJ, Pitt HA, et al. Tokyo Guidelines 2018: initial management of acute biliary infection and flowchart for acute cholangitis. J Hepatobil Pancreat Sci. 2018;25:31-40.

12. Haraldsson E, Lundell L, Swahn F, Enochsson L, Löhr JM, Arnelo U, et al. Results of an inter- and intraobserver agreement study. U Eur Gastroenterol J. 2017;5:504-10.

13. Freeman ML, Nelson DB, Sherman S, Haber GB, Herman ME, Dorsher PJ, et al. Complications of endoscopic biliary sphincterotomy. N Engl J Med. 1996;335:909-18.

14. Kiriyama S, Takada T, Strasberg SM, Solomkin JS, Mayumi T, Pitt HA, et al. New diagnostic criteria and severity assessment of acute cholangitis in revised Tokyo Guidelines. J Hepatobil Pancreat Sci. 2012;19:548-56.

15. Kuo CM, Chiu YC, Changchien CS, Tai WC, Chuah SK, Hu TH, et al. Endoscopic papillary balloon dilation for removal of bile duct stones: evaluation of outcomes and complications in 298 patients. $J$ Clin Gastroenterol. 2012;46:860-4.

16. Kuo CM, Chiu YC, Liang CM, Lu LS, Tai WC, Kuo YH, et al. Limited precut sphincterotomy combined with endoscopic papillary balloon dilation for common bile duct stone removal in patients with difficult biliary cannulation. BMC Gastroenterol. 2016;16:70.

17. Dumonceau JM, Andriulli A, Elmunzer BJ, Mariani A, Meister T, Deviere J, et al. Prophylaxis of postERCP pancreatitis: European Society of Gastrointestinal Endoscopy (ESGE) Guideline - updated June 2014. Endoscopy. 2014;46:799-815.

18. Park CH, Paik WH, Park ET, Shim CS, Lee TY, Kang C, et al. Aggressive intravenous hydration with Lactated Ringer's solution for prevention of post-ERCP pancreatitis: a prospective randomized multicenter clinical trial. Endoscopy. 2018;50:378-85.

19. Sugiyama M, Atomi Y. The benefits of endoscopic nasobiliary drainage without sphincterotomy for acute cholangitis. Am J Gastroenterol. 1998;93:2065-8.

20. Boender J, Nix GA, de Ridder MA, Dees J, Schütte HE, van Buuren HR, et al. Endoscopic sphincterotomy and biliary drainage in patients with cholangitis due to common bile duct stones. Am J Gastroenterol. 1995;90:233-8.

21. Sawas T, Arwani N, Al Halabi S, Vargo J. Sphincterotomy with endoscopic biliary drainage for severe acute cholangitis: a meta-analysis. Endosc Int Open. 2017;5:E103-9.

22. Hung TH, Tseng CW, Chen YC, Tseng KC, Hsieh YH, Tsai CC. Endoscopic papillary balloon dilation decreases the risk of bleeding in cirrhotic patients compared with endoscopic biliary sphincterotomy: A national population-based study. Med (Baltim). 2019;98:e16529.

23. Hui CK, Lai KC, Yuen MF, Ng M, Chan CK, Hu W, et al. Does the addition of endoscopic sphincterotomy to stent insertion improve drainage of the bile duct in acute suppurative cholangitis? Gastrointest Endosc. 2003;58:500-4. 
24. Wiggins T, Markar SR, MacKenzie H, Faiz O, Mukherjee D, Khoo DE, et al. Optimum timing of emergency cholecystectomy for acute cholecystitis in England: population-based cohort study. Surg Endosc. 2019;33:2495-502.

25. Polo M, Duclos A, Polazzi S, Payet C, Lifante JC, Cotte E, et al. Acute cholecystitis-optimal timing for early cholecystectomy: a French Nationwide Study. J Gastrointest Surg. 2015;19:2003-10.

26. Zafar SN, Obirieze A, Adesibikan B, Cornwell EE, Fullum TM, Tran DD. Optimal time for early laparoscopic cholecystectomy for acute cholecystitis. JAMA Surg. 2015;150:129-36.

27. Jansen B, Peters G. Foreign body associated infection. J Antimicrob Chemother. 1993;32(Suppl A):69-75.

28. Navaneethan U, Jayanthi V, Mohan P. Pathogenesis of cholangitis in obstructive jaundice-revisited. Minerva Gastroenterol Dietol. 2011;57:97-104.

29. Sharma G, Sharma S, Sharma P, Chandola D, Dang S, Gupta S, et al. Escherichia coli biofilm: development and therapeutic strategies. J Appl Microbiol. 2016;121:309-19.

30. Nirwati H, Sinanjung K, Fahrunissa F, Wijaya F, Napitupulu S, Hati VP, et al. Biofilm formation and antibiotic resistance of Klebsiella pneumoniae isolated from clinical samples in a tertiary care hospital, Klaten, Indonesia. BMC Proc. 2019;13(Suppl 11);Suppl 11:20.

31. Kim H, Ryu JH, Beuchat LR. Attachment of and biofilm formation by Enterobacter sakazakii on stainless steel and enteral feeding tubes. Appl Environ Microbiol. 2006;72:5846-56.

32. Mohamed JA, Huang DB. Biofilm formation by enterococci. J Med Microbiol. 2007;56:1581-8.

33. Swidsinski A, Schlien P, Pernthaler A, Gottschalk U, Bärlehner E, Decker G, et al. Bacterial biofilm within diseased pancreatic and biliary tracts. Gut. 2005;54:388-95.

34. Kim HJ, Choi HS, Park JH, Park DI, Cho YK, Sohn Cl, et al. Factors influencing the technical difficulty of endoscopic clearance of bile duct stones. Gastrointest Endosc. 2007;66:1154-60.

35. Kuo CM, Chiu YC, Liang CM, Wu CK, Lu LS, Tai WC, et al. The efficacy of limited endoscopic sphincterotomy plus endoscopic papillary large balloon dilation for removal of large bile duct stones. BMC Gastroenterol. 2019;19:93.

\section{Figures}




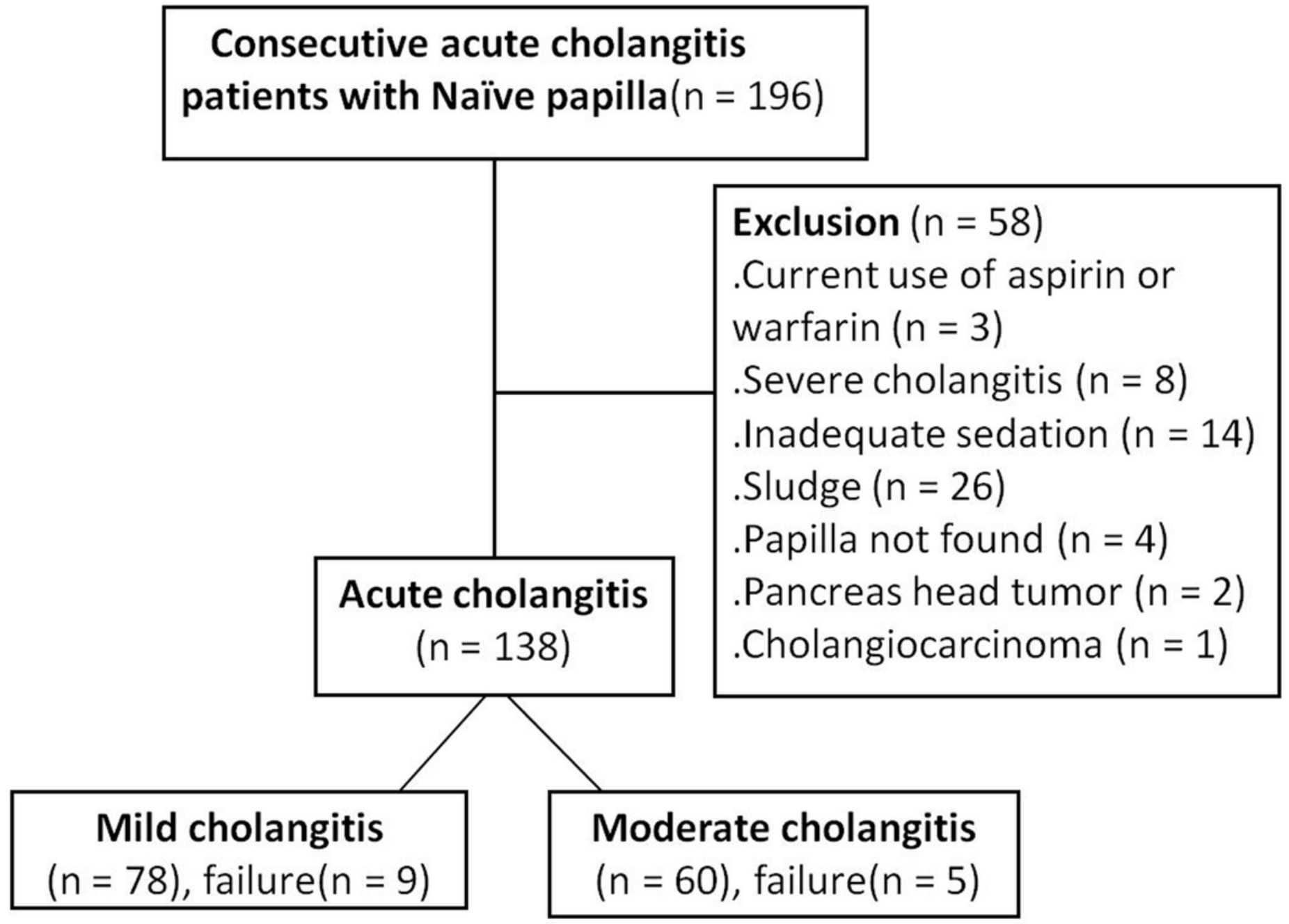

Figure 1

The flow diagram of the prospective enrollment of consecutive patients with mild/moderate acute cholangitis. 


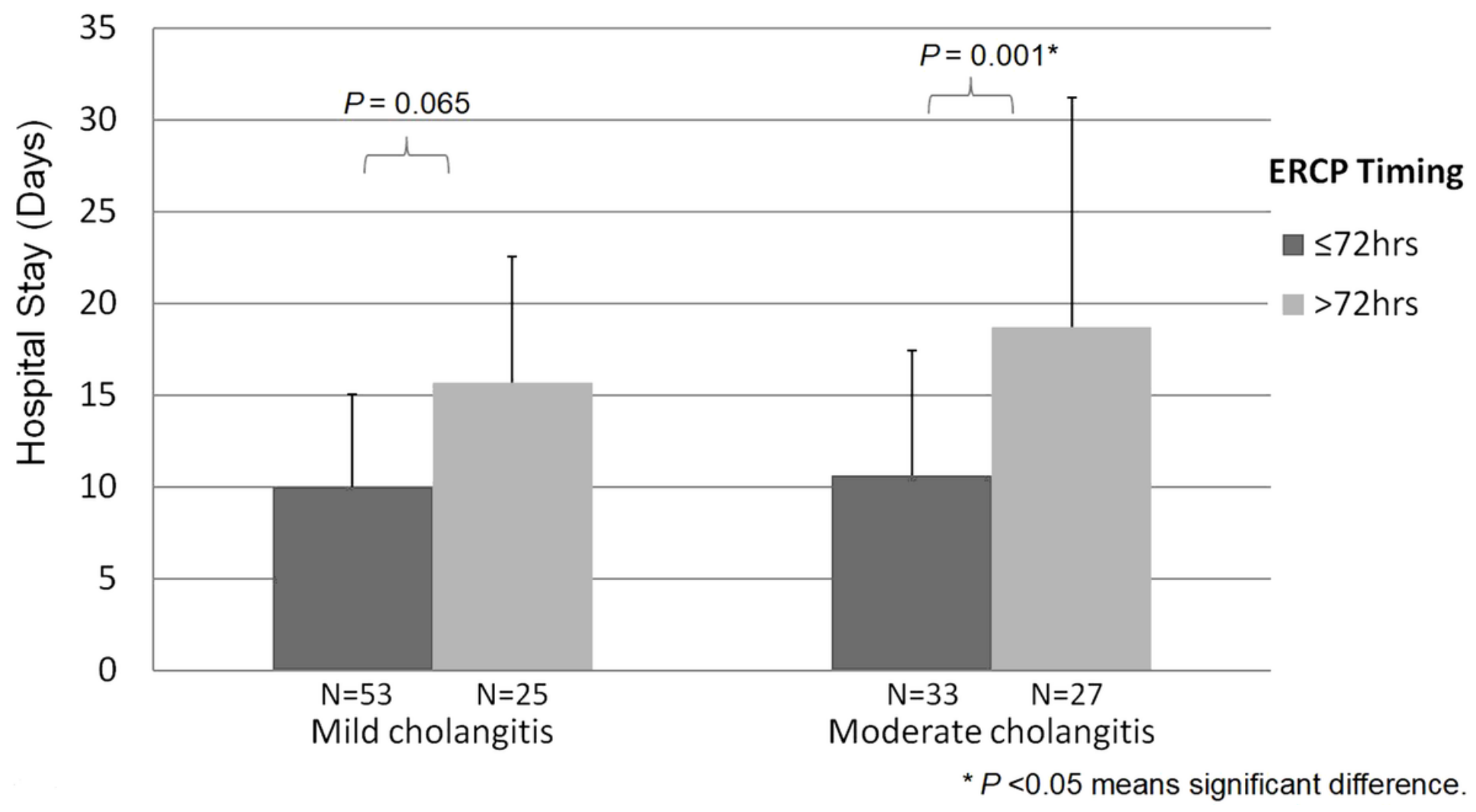

Figure 2

Relationship between the length of hospitalization and single-stage ERCP timing. 


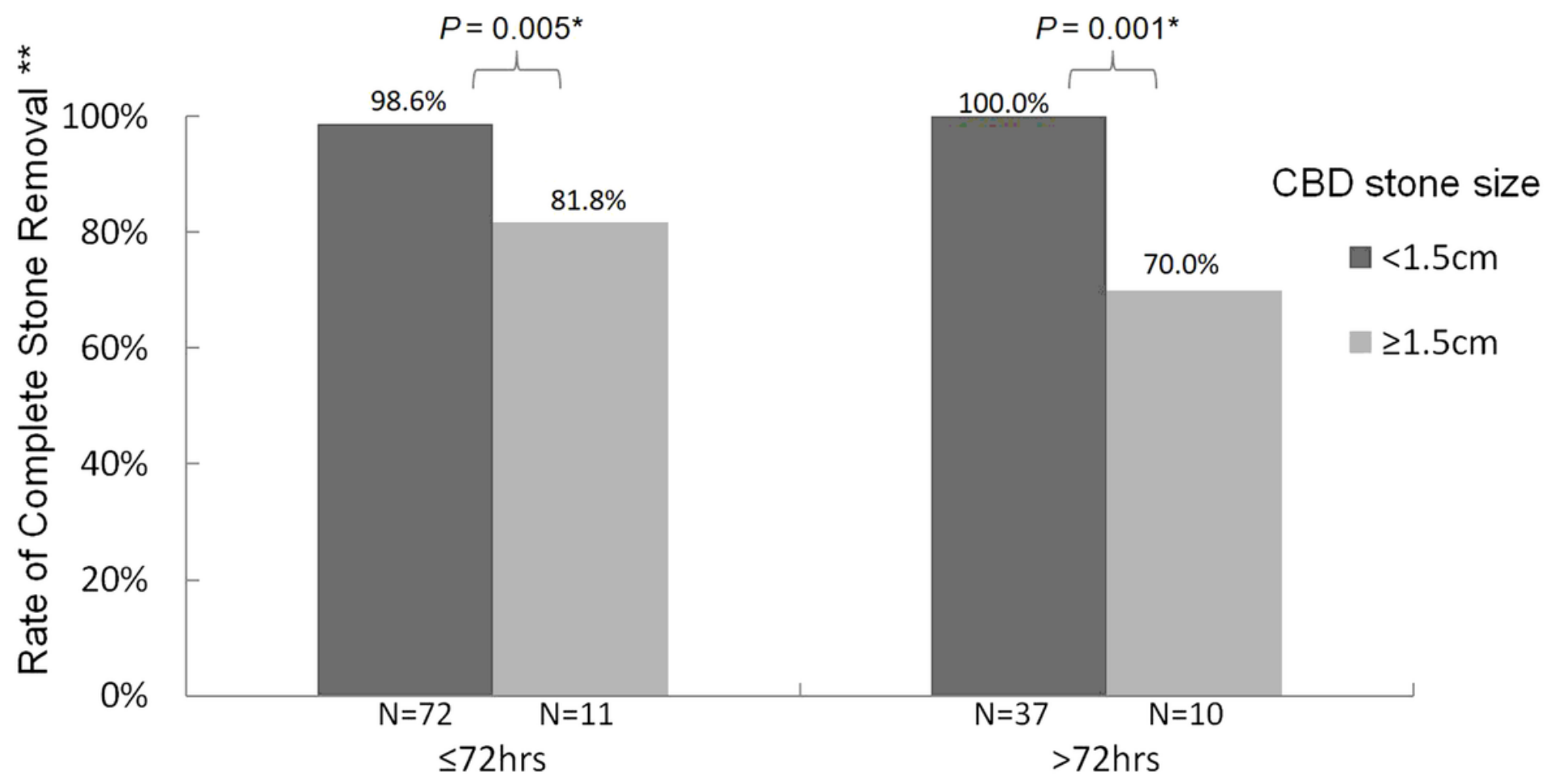

ERCP Timing from ER $\quad{ }^{*} P<0.05$ means significant difference. ** Excluding the cases of cannulation failure

\section{Figure 3}

Relationship between bile duct stone size and stone removal success rate after excluding cannulation failure cases. 